\title{
Evaluation of linkage disequilibrium and its effect on non-parametric multipoint linkage analysis using two high density single-nucleotide polymorphism mapping panels Sarah Shaw Murray*
}

\author{
Address: Illumina, Inc., 9885 Towne Centre Drive, San Diego, CA 92121, USA \\ Email: Sarah Shaw Murray* - smurray@illumina.com \\ * Corresponding author
}

\author{
from Genetic Analysis Workshop I4: Microsatellite and single-nucleotide polymorphism \\ Noordwijkerhout, The Netherlands, 7-10 September 2004 \\ Published: 30 December 2005 \\ BMC Genetics 2005, 6(Suppl I):S85 doi:I0.I I86/I47I-2I56-6-SI-S85
}

\begin{abstract}
Genotype data from the Illumina Linkage III SNP panel ( $n=4,720$ SNPs) and the Affymetrix $10 \mathrm{k}$ mapping array ( $n=11,120$ SNPs) were used to test the effects of linkage disequilibrium (LD) between SNPs in a linkage analysis in the Collaborative Study on the Genetics of Alcoholism pedigree collection ( 143 pedigrees; I,6I4 individuals). The average $r^{2}$ between adjacent markers across the genetic map was $0.099 \pm 0.003$ in the Illumina III panel and $0.17 \pm 0.003$ in the Affymetrix $10 \mathrm{k}$ array. In order to determine the effect of LD between marker loci in a nonparametric multipoint linkage analysis, markers in strong LD with another marker $\left(r^{2}>0.40\right)$ were removed $(n$ $=47 \mathrm{I}$ loci in the Illumina panel; $n=1,804$ loci in the Affymetrix panel) and the linkage analysis results were compared to the results using the entire marker sets. In all analyses using the ALDXI phenotype, 8 linkage regions on 5 chromosomes $(2,7,10,11, X)$ were detected (peak markers $p$ $<0.0 \mathrm{I}$ ), and the Illumina panel detected an additional region on chromosome 6 . Analysis of the same pedigree set and ALDXI phenotype using short tandem repeat markers (STRs) resulted in 3 linkage regions on 3 chromosomes (peak markers $p<0.01$ ). These results suggest that in this pedigree set, LD between loci with spacing similar to the SNP panels tested may not significantly affect the overall detection of linkage regions in a genome scan. Moreover, since the data quality and information content are greatly improved in the SNP panels over STR genotyping methods, new linkage regions may be identified due to higher information content and data quality in a dense SNP linkage panel.
\end{abstract}

\section{Background}

For many years short tandem repeat (STR) or microsatellite markers have been used as the standard genetic markers for linkage mapping [1-5]. STRs typically have a high degree of heterozygosity and provide high information content per marker [6]. They occur widely throughout the genome, with over 5,000 STRs being mapped to a highresolution genetic map [5]. However, a drawback to using microsatellite markers is that because of the large number of alleles differing by as few as 2 to $4 \mathrm{bp}$, analysis has required electrophoretic separation. Because of this technical limitation, their analysis is time consuming and not amenable to highly multiplexed, automated formats.

In contrast, single-nucleotide polymorphism (SNP) assays are more amenable to multiplexing and are easier to automate, which increases accuracy and reliability. Therefore, more complete linkage studies with thousands of markers, and studies with large numbers of DNA samples are more feasible using SNPs. In addition, there is an 
abundance of SNPs: currently over 8 million human SNPs are in public databases, many of which have been validated http://www.ncbi.nlm.nih.gov/SNP/. Recent reports have shown SNP linkage panels have higher information content and data quality than STR marker panels [7-9]. However, an important consideration for using numerous closely spaced SNPs in a linkage analysis is the analytical method used for statistical analysis of the genotype data. Genotyping many SNPs at high density compensates for the fact that each individual SNP has limited heterozygosity (maximum theoretical heterozygosity $=50 \%$ ). Therefore, in order to maximize the information content (IC), one must use multipoint linkage analysis across a map of densely spaced SNPs or use multi-SNP haplotypes in a two-point linkage analysis. Another important consideration is the amount of linkage disequilibrium (LD) that exists between loci when using a mapping panel of densely spaced SNPs in a linkage analysis. Existing linkage analysis programs assume linkage equilibrium between loci. This assumption is violated if $\mathrm{LD}$ exists between SNP loci and may introduce a bias in the resulting LOD score. This report describes the results of determining the amount of LD that exists between loci in two different high-density SNP linkage panels and their effect on the overall linkage analysis results using 143 Collaborative Study on the Genetics of Alcoholism (COGA) pedigrees segregating the ALDX1 phenotype.

\section{Methods \\ Samples}

One hundred forty-three pedigrees from the COGA study comprised 1,614 individuals (1,332 genotyped individuals) segregating alcoholism were used in all analyses. The 143 pedigrees had 256 sibships. Of these 256 sibships, $123(48 \%)$ had both parents, 75 (29\%) had 1 parent, and $58(23 \%)$ had no parents available for SNP genotyping. The majority of the individuals had a self-reported ethnicity of White, non-Hispanic $(n=1,074)$. The next largest self-reported ethnic group was black, non-Hispanic ( $n=$ 191).

\section{Genetic markers}

The Illumina Linkage III SNP panel and the Affymetrix 10 $\mathrm{k}$ mapping array were used for both LD calculations and linkage analyses. The Illumina panel used for the Genetic Analysis Workshop 14 study had 4,720 SNP loci with a mean and median physical spacing of $615 \mathrm{~kb}$ and $406 \mathrm{~kb}$, respectively. The data were of exceptional quality with a high call rate $(99.95 \%)$, low error rate $(0.005 \%)$, and low rate of Mendelian inconsistencies $(0.09 \%)$. From a genetic map generated from $28 \mathrm{CEPH}$ pedigrees [9], the mean and median genetic spacing was $1.5 \mathrm{cM}$ and $1.1 \mathrm{cM}$, respectively. The Affymetrix $10 \mathrm{k}$ mapping array had 11,120 loci with a mean and median physical spacing of $210 \mathrm{~kb}$ and $105 \mathrm{~kb}$, respectively. The data were also of high quality with a $94.75 \%$ call rate, $0.052 \%$ error rate, and $0.12 \%$ rate of Mendelian inconsistencies. From a genetic map derived from linear interpolation of physical map position and a high resolution microsatellite genetic map, the mean genetic spacing was $0.32 \mathrm{cM}$ [8]. The COGA STR marker set had 328 markers with an average genetic spacing of $10.45 \mathrm{cM}$. Previously the data quality for the COGA STR dataset were reported [10], and the missing genotype rate and Mendelian inconsistency rate were grouped together and estimated to be $4 \%$. The error rate was estimated to be $0.8 \%$.

\section{Evaluation of inter-marker LD}

Inter-marker LD was determined from between all SNP loci in the two SNP panels, respectively, in 244 unrelated individuals. LD strength was derived from estimated haplotypes using the expectation-maximization algorithm of Slatkin and Excoffier [11] as implemented in the ldmax component of the program GOLD [12]. LD strength, as measured by the parameter $r^{2}$, was determined for each pair-wise combination. In particular, LD between each adjacent marker on the genetic map used for linkage analysis was investigated. Loci in strong LD with another marker $\left(r^{2}>0.40\right)$ were removed and the linkage analysis was repeated using the subset of markers where all loci have no or weak LD $\left(r^{2}<0.40\right)$.

\section{Multipoint nonparametric linkage (NPL) analysis}

Linkage analysis of both SNP panels and the COGA STR panel were analyzed using the ALDX1 phenotype. Genotype data were analyzed using multipoint nonparametric methods as implemented in GENEHUNTER v2.1 [13] on all pedigrees using the map distances provided with the genotype data. For the SNP loci, because the markers are densely mapped, multipoint analyses were carried out using one step between each marker. For the STR loci, multipoint analyses were carried out using four steps between each marker. Peak loci were determined as those regions with $p$-values $<0.01$.

\section{Results}

Inter-marker LD

LD strength $\left(r^{2}\right)$ between each adjacent marker pair on the genetic map was evaluated in both SNP panels. For the Illumina SNP panel, the average $r^{2}$ between each adjacent marker pair on the genetic map was $0.099 \pm 0.003(\mathrm{SD}=$ 0.23 ). As shown in Figure $1 \mathrm{a},>75 \%$ of all marker pairs had an $r^{2}<0.05$. Figure $1 \mathrm{~b}$ shows the proportion of marker pairs that have $r^{2}>0.4$ by chromosome. The $\mathrm{X}$ chromosome had the highest proportion of marker pairs with $r^{2}>0.4$ (33/123 marker pairs; 27\%). Overall, 471/ 4,697 marker pairs $(10 \%)$ had $r^{2}>0.4$. One marker from each of the 471 marker pairs with $r^{2}>0.40$ was randomly removed. In the case where there were multiple adjacent SNPs with $r^{2}>0.40$, the minimum numbers of loci were 

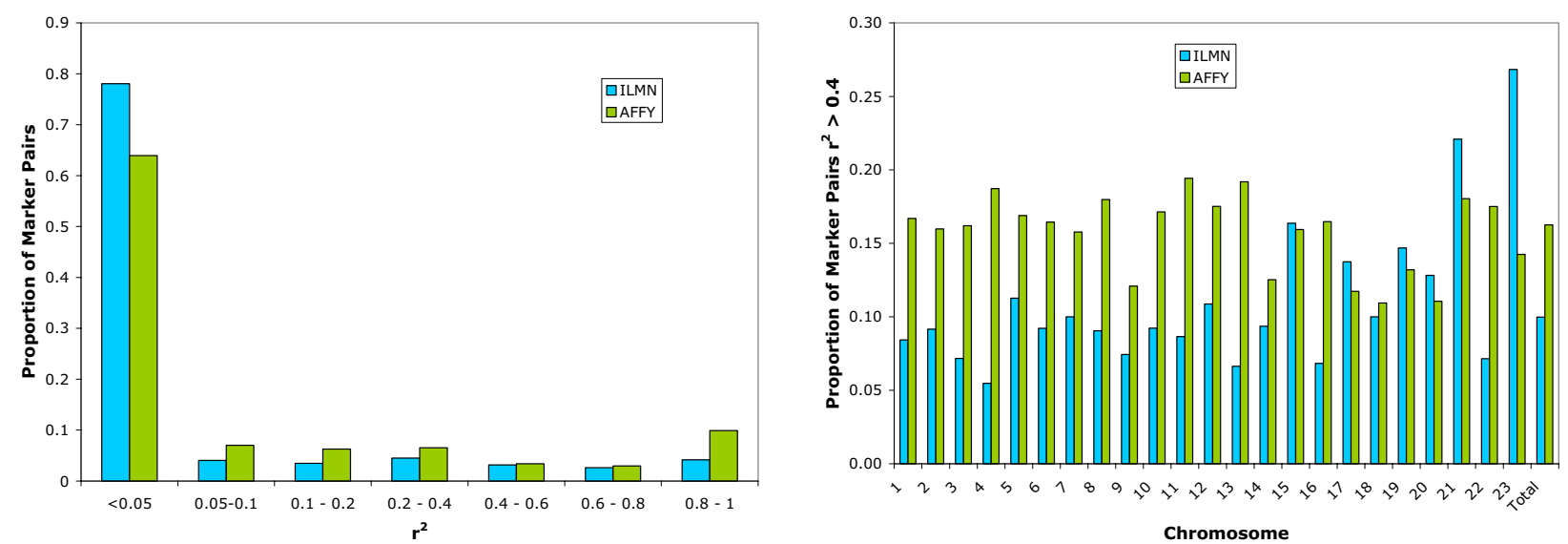

Figure I

LD strength in the Illumina Linkage III SNP panel (ILMN) and the Affymetrix $10 \mathrm{k}$ mapping array (AFFY). a) Distribution of marker pairs by LD strength $\left(r^{2}\right)$; b) Proportion of marker pairs $r^{2}>0.4$ by chromosome (chromosome $23=X$ chromosome).
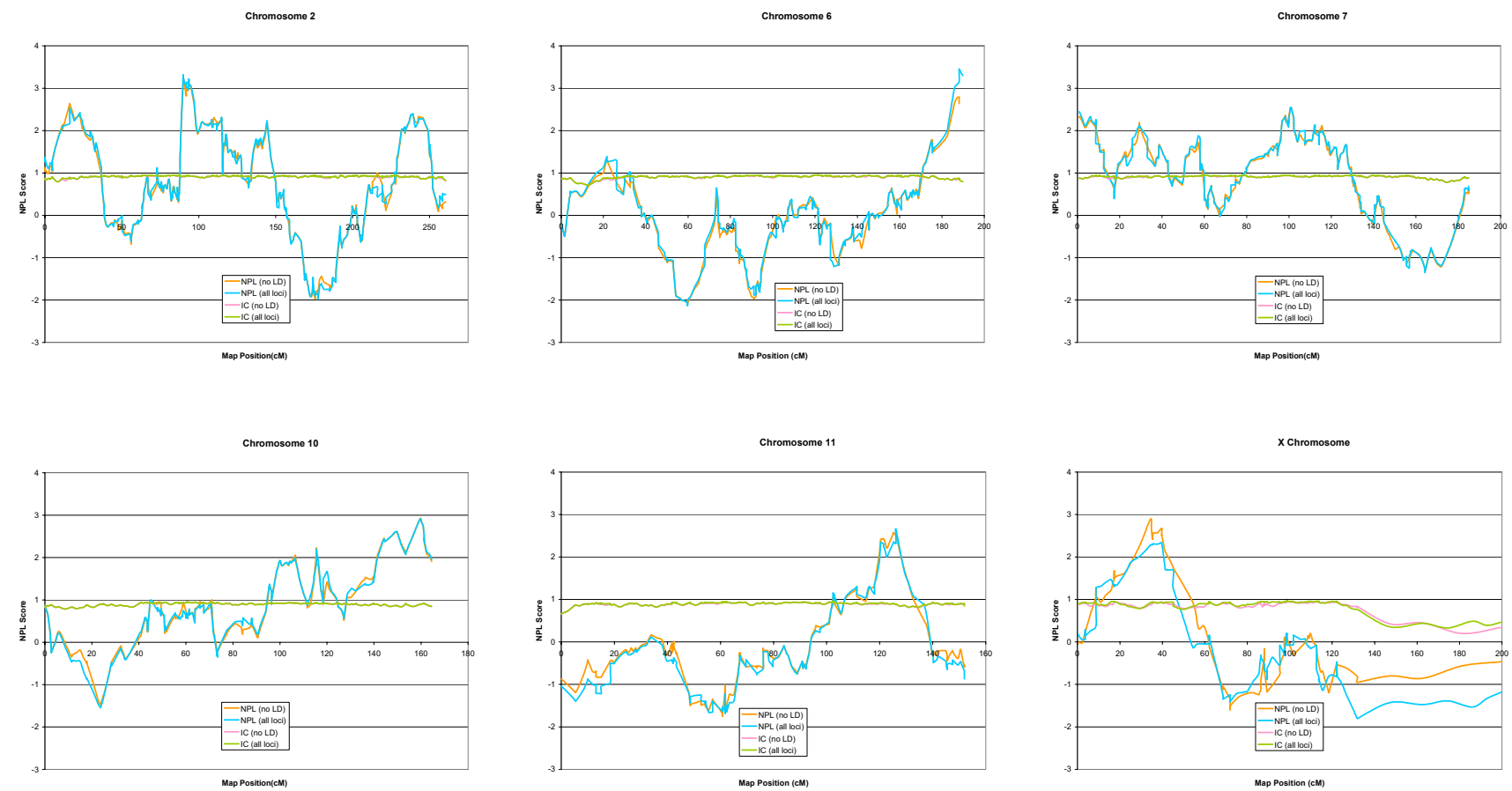

\section{Figure 2}

Multipoint NPL analysis of ALDXI phenotype using the Illumina III SNP linkage panel. Multipoint NPL scores across the entire chromosome for all SNPs (blue line) and a subset of SNPs with weak or no LD (orange line) are shown for the six chromosomes that have regions with corresponding $\mathrm{P}$-values $<0.0 \mathrm{I}$. In addition, the information content (IC) curves are also shown for all loci (green line) and the subset of SNPs with weak or no LD (pink line).

removed so that the remaining adjacent loci had $r^{2}<0.40$. 
Table I: Linkage results using the Illumina Linkage III SNP Panel and the Affymetrix $10 \mathrm{k}$ Mapping Array.

\begin{tabular}{|c|c|c|c|c|c|c|c|c|c|c|c|c|}
\hline \multirow[b]{2}{*}{ Chr } & \multicolumn{6}{|c|}{ Illumina III SNP linkage panel } & \multicolumn{6}{|c|}{ Affymetrix $10 \mathrm{k}$ mapping array } \\
\hline & Marker & $\begin{array}{l}\text { Position } \\
\text { (cM) }\end{array}$ & Build34 bp & NPL & $p$-value & IC & Marker & $\begin{array}{l}\text { Position } \\
\text { (cM) }\end{array}$ & Build34 bp & NPL & $p$-value & IC \\
\hline $2 p 25$ & rs1025053 & 16.1 & $8238 \mid 43$ & 2.53 & 0.0073 & 0.89 & tsc004I 232 & 5.74 & 2888794 & 2.71 & 0.0047 & 0.91 \\
\hline $2 p 25$ & rs1025053 & 16.1 & 8238143 & 2.64 & 0.0056 & 0.90 & tsc004I 232 & 5.74 & 2888794 & 2.43 & 0.0095 & 0.91 \\
\hline $2 p \mid 4$ & rs 1000756 & 89.9 & 66769368 & 3.31 & 0.00090 & 0.93 & tsc 1090282 & 90.58 & 67905612 & 3.82 & 0.00019 & 0.94 \\
\hline $2 p \mid 4$ & rsll 06577 & 89.9 & 67023129 & 3.26 & 0.0010 & 0.93 & tscl090282 & 90.58 & 67905612 & 3.80 & 0.00021 & 0.94 \\
\hline $2 q 37$ & rs|453867 & 239.2 & $233 \mid 20438$ & 2.39 & 0.010 & 0.93 & tsc0524987 & 244.44 & 236010878 & 2.71 & 0.0046 & 0.89 \\
\hline $2 q 37$ & rs|453867 & 239.2 & 233120438 & 2.38 & 0.010 & 0.93 & tsc0524987 & 244.44 & 236010878 & 2.68 & 0.0051 & 0.89 \\
\hline $6 q 27$ & rs760909 & | 88.| & 170713389 & 3.46 & 0.00058 & 0.86 & tsc0050465 & 185.42 & multiple pos. & 1.85 & 0.035 & 0.81 \\
\hline $6 q 27$ & rs727619 & 188.1 & 170562703 & 2.79 & 0.0037 & 0.88 & tsc0050465 & 185.42 & multiple pos. & 1.77 & 0.041 & 0.81 \\
\hline 7p22-2I & rsl057558 & 0 & 806704 & 2.62 & 0.0058 & 0.89 & tscI592599 & 31.68 & 17610703 & 2.55 & 0.0070 & 0.94 \\
\hline $7 p 22-21$ & rs|l|27460 & 0 & 623105 & 2.35 & 0.011 & 0.89 & tsc092500I & 31.35 & 17305065 & 2.42 & 0.0096 & 0.93 \\
\hline $7 q 21$ & rs1023564 & 101.1 & 88453743 & 2.54 & 0.007 I & 0.94 & tsc004927 I & 101.05 & $89624 I I 5$ & 2.77 & 0.0039 & 0.94 \\
\hline $7 q 21$ & rs717474 & 101.1 & 88403326 & 2.54 & 0.0072 & 0.94 & tscl 240889 & 99.05 & 85964828 & 2.82 & 0.0035 & 0.94 \\
\hline $10 q 26$ & rs|536087 & 159.6 & 131394560 & 2.90 & 0.0028 & 0.90 & tsc0960887 & $|7| .4 \mid$ & | 32054567 & 3.25 & 0.0011 & 0.88 \\
\hline $10 q 26$ & rsl536087 & 159.6 & 131394560 & 2.92 & 0.0027 & 0.90 & tsc0960887 & $|7| .4 \mid$ & | 32054567 & 3.03 & 0.0020 & 0.87 \\
\hline I Iq23 & rs6I 7847 & | 26.| & | 20940295 & 2.65 & 0.0054 & 0.90 & tsc00535 I 2 & 125.27 & 121196164 & 2.82 & 0.0035 & 0.92 \\
\hline $11 \mathrm{lq} 23$ & rs575030 & 125.1 & 120630050 & 2.57 & 0.0066 & 0.91 & tsc005I 264 & 125.15 & $121 \mid 12747$ & 2.51 & 0.0076 & 0.92 \\
\hline Xp22 & rs22503I & 39.7 & 28452585 & 2.34 & 0.011 & 0.93 & tsc0038428 & 42.78 & 25961925 & 2.55 & 0.007 I & 0.97 \\
\hline Xp22 & rs795489 & 34.4 & 23062119 & 2.89 & 0.0030 & 0.88 & tsc0038428 & 42.78 & 25961925 & 2.68 & 0.0053 & 0.94 \\
\hline
\end{tabular}

aPeak loci $p<0.0$ I for all loci (bold) and loci $r^{2}<0.40$ (not bold)

A subset of 4,149 loci was used for subsequent linkage analyses where all loci had weak or no LD. For the Affymetrix SNP panel, the average LD between loci was higher (as expected) because the SNPs are more closely spaced. The average $r^{2}$ between each adjacent marker pair on the genetic map was $0.17 \pm 0.003(\mathrm{SD}=0.30)$, and less than $65 \%$ of all marker pairs had an $r^{2}<0.05$ (Figure 1a). There were 1,804 marker pairs with $r^{2}>0.40$. One marker from each pair was removed for subsequent linkage analysis using the remaining 9,316 loci with weak or no LD.

\section{NPL multipoint analysis}

Genotype data were analyzed using the ALDX1 phenotype and the NPL multipoint analysis methods as implemented in the program GENEHUNTER v2.1 [12]. For the complete set of 4,720 SNP loci in the Illumina SNP panel, nine regions on six chromosomes (chromosomes 2, 6, 7, $10,11, \mathrm{X}$ ) had peak NPL scores with corresponding $p$-values $<0.01$ and are summarized in Table 1 . Next, a subset of the Illumina SNP panel was analyzed where all 4,149 loci had weak or no LD $\left(r^{2}<0.40\right)$. The same nine regions on six chromosomes were identified in this analysis. These results are also shown in Table 1 . The biggest difference in results between the two analyses (all loci versus loci with no or weak LD) occurred on the X chromosome, where a high proportion of loci $(17 \%)$ were removed and the IC at the linkage peak dropped from 0.93 to 0.88 . The linkage peak shifted approximately $5 \mathrm{cM}$ between the two analyses. Another big difference between the two analyses occurred on chromosome 6, where 3 of the 9 loci in the interval were removed, including the peak marker from the analysis of all loci. The analysis using a subset of markers was still significant $(p=0.004)$, though less significant than the analysis with all loci $(p=0.0006)$. Even though there were moderate differences between the two analyses on these two chromosomes, the same regions were identified in both analyses with $p$-values $\leq 0.01$. The six chromosomes with $p$-values $\leq 0.01$ are shown in Figure 2. For the complete set of 11,120 loci in the Affymetrix $10 \mathrm{k}$ Mapping Array, eight regions on five chromosomes (chromosomes 2, 7, 10, 11, X) had peak NPL scores with corresponding $p$-values $<0.01$ as shown in Table 1 . A second analysis without the 1,804 loci in strong LD resulted in the same eight regions and similar NPL scores and $p$-values (Table 1). The Affymetrix SNP panel did detect a peak at the same region on chromosome 6 as in the Illumina SNP panel, however the $p$-value was $>0.01$ (Table 1, Figure 3). The five chromosomes with linkage regions $(p<$ 0.01 ) are shown in Figure 3.

IC was also compared across all marker sets as shown in Table 2. The genome-wide average IC for the Illumina set was $0.89(\min =0.33, \max =0.97)$ with only one chromosome having an average IC $<0.80$. In addition, the IC was consistently high across most of the genome (with the exception of a gap in Xq23-q28), with 2 chromosomes having a minimum IC less than 0.70 . The genome-wide average IC of the Affymetrix set was slightly higher (average IC $=0.90$, range $=0.43-0.97$ ) with all chromosomes having an average IC $>0.80$. Even though this panel had 

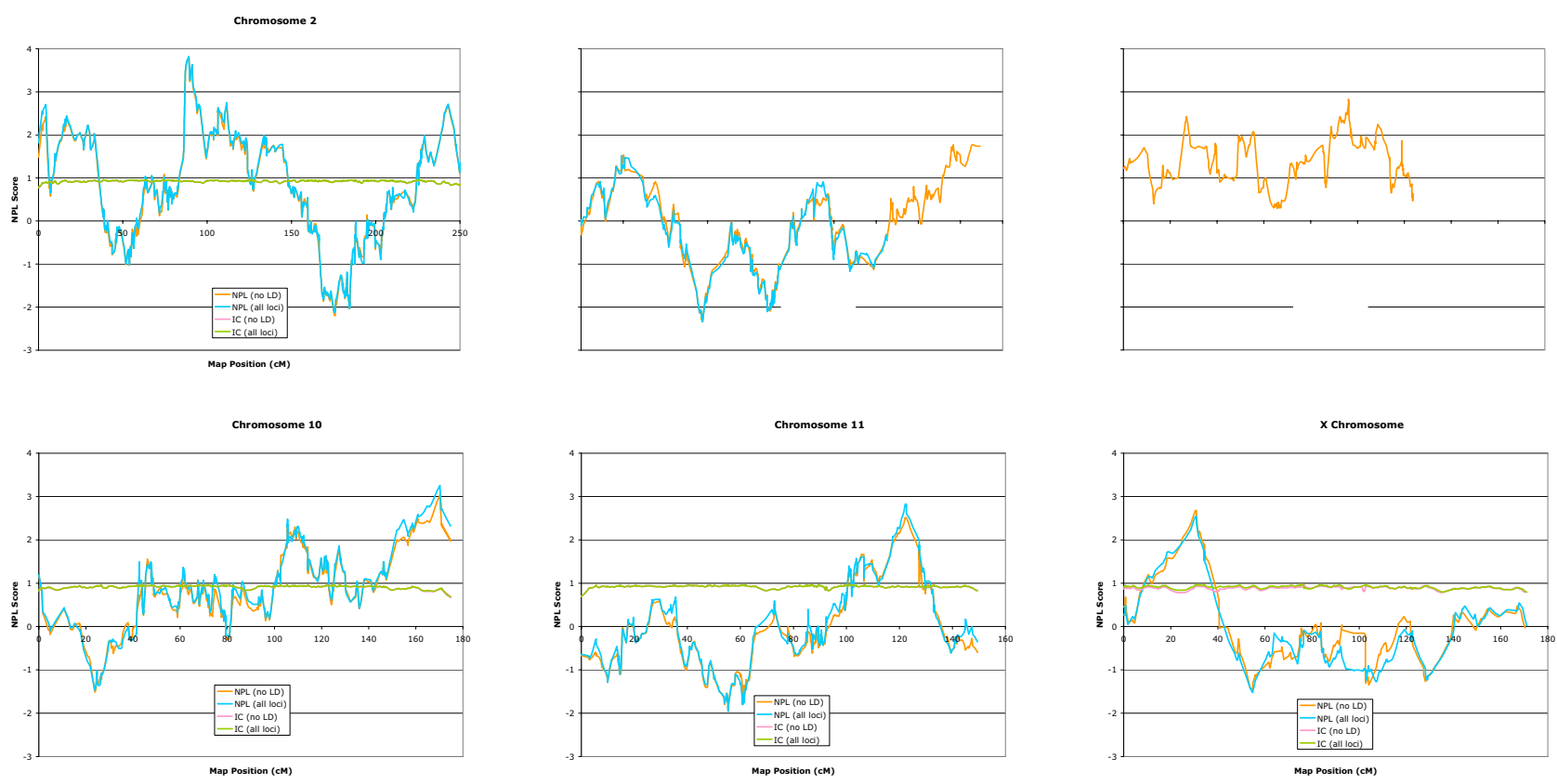

\section{Figure 3}

Multipoint NPL analysis of ALDXI phenotype using the Affymetrix 10 k Mapping Array. Multipoint NPL scores across the entire chromosome for all SNPs (blue line) and a subset of SNPs with weak or no LD (orange line) are shown for six chromosomes. Chromosomes 2, 7, 10, II, and X have regions with corresponding $p$-values < 0.01 ; chromosome 6 is shown for comparison with the Illumina SNP mapping panel results. In addition, the information content (IC) curves are also shown for all loci (green line) and the subset of SNPs with weak or no LD (pink line).

over twice the number of loci, there were only modest improvements in average IC, and actually there were more regions of lower IC, since 8 chromosomes had a minimum IC $<0.70$.

Finally, STR data were analyzed using the ALDX1 phenotype. Two of the 9 linkage peaks detected using the highdensity SNP map were detected with STRs at $p<0.01$. The resulting peak loci and linkage intervals are summarized in Table 3. The peak on chromosome 7 does not overlap with the two peaks on chromosome 7 detected using high density SNPs, however it is flanked by the two peaks detected in the SNP panels. As shown in Table 2, the genome-wide IC was considerably lower than both SNP panels (average $=0.61$ ), since no chromosomes had an average IC $>0.80$. In addition, all chromosomes had minimum IC $<0.70$.

\section{Discussion}

An early study suggested that if LD exists between a trait and marker and if LD is not taken into account in a linkage analysis, the resulting LOD score would be reduced [14]. However, LD that exists between two marker loci may not necessarily have the same bias, and could in fact artificially increase a LOD score in a linkage analysis. For example, if haplotype frequencies are calculated assuming linkage equilibrium when in fact LD exists and the linked haplotype frequency is underestimated, then this could artificially inflate the resulting LOD score [15]. This effect would be largest in pedigrees in which genotypes are not available for founding individuals. In this study using the Illumina SNP panel, a low level of LD was detected between each SNP overall, and 78\% of all marker pairs in order of the genetic map had an $r^{2}<0.05$. Using the Affymetrix mapping array, a higher proportion of marker pairs had high LD genome-wide, and 65\% of all marker pairs had an $r^{2}<0.05$.

Overall, in both SNP panels there was very little difference between results using the entire marker set and the reduced marker set without loci in strong LD. Using both SNP panels, the same regions were detected in both analyses at $p<0.01$, with modest changes in $p$-values and NPL scores. The NPL scores and $p$-values did not consistently become more or less significant with the removal of loci in $\mathrm{LD}$. In addition, there was high concordance in terms of significance and location of linkage peaks between the two SNP mapping panels. The main difference in findings between the two panels was that the Affymetrix mapping array did not detect the linkage peak at $p<0.01$ on chro- 
Table 2: Information content comparisons in the two SNP marker sets and the COGA STR marker set (Average (min, max))

\begin{tabular}{|c|c|c|c|c|c|}
\hline \multirow[t]{2}{*}{ Chromosome } & \multicolumn{2}{|c|}{ Illumina } & \multicolumn{2}{|c|}{ Affymetrix } & \multirow[t]{2}{*}{ COGA STR (all loci) } \\
\hline & all loci & $r^{2}<0.4$ & all loci & $r^{2}<0.4$ & \\
\hline I & $0.91(0.75,0.95)$ & $0.91(0.75,0.96)$ & $0.91(0.69,0.95)$ & $0.91(0.69,0.95)$ & $0.64(0.28,0.90)$ \\
\hline 2 & $0.91(0.79,0.95)$ & $0.91(0.79,0.95)$ & $0.92(0.78,0.96)$ & $0.92(0.77,0.96)$ & $0.70(0.47,0.90)$ \\
\hline 3 & $0.91(0.82,0.95)$ & $0.91(0.83,0.95)$ & $0.92(0.84,0.97)$ & $0.92(0.83,0.97)$ & $0.59(0.41,0.86)$ \\
\hline 4 & $0.89(0.72,0.96)$ & $0.89(0.72,0.95)$ & $0.91(0.59,0.96)$ & $0.91(0.59,0.96)$ & $0.62(0.47,0.78)$ \\
\hline 5 & $0.90(0.77,0.95)$ & $0.90(0.77,0.95)$ & $0.92(0.75,0.96)$ & $0.92(0.75,0.96)$ & $0.63(0.37,0.85)$ \\
\hline 6 & $0.90(0.72,0.96)$ & $0.90(0.71,0.95)$ & $0.92(0.71,0.96)$ & $0.92(0.71,0.96)$ & $0.60(0.37,0.84)$ \\
\hline 7 & $0.91(0.78,0.95)$ & $0.91(0.78,0.95)$ & $0.91(0.78,0.96)$ & $0.91(0.77,0.95)$ & $0.78(0.59,0.92)$ \\
\hline 8 & $0.90(0.79,0.95)$ & $0.89(0.75,0.95)$ & $0.91(0.75,0.95)$ & $0.91(0.75,0.95)$ & $0.67(0.44,0.87)$ \\
\hline 9 & $0.89(0.78,0.95)$ & $0.89(0.77,0.95)$ & $0.91(0.70,0.96)$ & $0.91(0.69,0.96)$ & $0.62(0.47,0.85)$ \\
\hline 10 & $0.89(0.78,0.95)$ & $0.89(0.78,0.95)$ & $0.91(0.68,0.95)$ & $0.91(0.68,0.95)$ & $0.48(0.31,0.76)$ \\
\hline II & $0.89(0.66,0.95)$ & $0.89(0.66,0.95)$ & $0.92(0.70,0.96)$ & $0.92(0.69,0.96)$ & $0.51(0.30,0.74)$ \\
\hline 12 & $0.90(0.75,0.95)$ & $0.89(0.75,0.95)$ & $0.90(0.74,0.96)$ & $0.90(0.74,0.96)$ & $0.65(0.38,0.90)$ \\
\hline 13 & $0.90(0.77,0.95)$ & $0.89(0.76,0.95)$ & $0.91(0.62,0.96)$ & $0.91(0.62,0.96)$ & $0.62(0.33,0.86)$ \\
\hline 14 & $0.90(0.79,0.95)$ & $0.89(0.79,0.95)$ & $0.86(0.43,0.95)$ & $0.86(0.42,0.95)$ & $0.62(0.49,0.79)$ \\
\hline 15 & $0.89(0.77,0.95)$ & $0.89(0.76,0.95)$ & $0.90(0.83,0.95)$ & $0.90(0.83,0.95)$ & $0.67(0.56,0.85)$ \\
\hline 16 & $0.90(0.75,0.95)$ & $0.89(0.74,0.95)$ & $0.87(0.46,0.95)$ & $0.86(0.46,0.95)$ & $0.57(0.33,0.82)$ \\
\hline 17 & $0.89(0.79,0.94)$ & $0.88(0.78,0.94)$ & $0.85(0.51,0.94)$ & $0.85(0.51,0.94)$ & $0.56(0.35,0.77)$ \\
\hline 18 & $0.88(0.75,0.95)$ & $0.88(0.75,0.95)$ & $0.91(0.70,0.95)$ & $0.91(0.70,0.95)$ & $0.51(0.35,0.69)$ \\
\hline 19 & $0.88(0.73,0.95)$ & $0.86(0.71,0.94)$ & $0.81(0.57,0.94)$ & $0.81(0.56,0.94)$ & $0.56(0.4 I, 0.76)$ \\
\hline 20 & $0.89(0.82,0.94)$ & $0.89(0.80,0.94)$ & $0.89(0.78,0.95)$ & $0.89(0.71,0.95)$ & $0.60(0.39,0.82)$ \\
\hline 21 & $0.91(0.85,0.94)$ & $0.90(0.84,0.94)$ & $0.92(0.75,0.95)$ & $0.91(0.75,0.95)$ & $0.59(0.39,0.83)$ \\
\hline 22 & $0.89(0.79,0.95)$ & $0.88(0.78,0.95)$ & $0.84(0.77,0.94)$ & $0.84(0.77,0.94)$ & $0.55(0.34,0.76)$ \\
\hline $\mathrm{X}$ & $0.74(0.33,0.97)$ & $0.72(0.20,0.97)$ & $0.91(0.84,0.97)$ & $0.88(0.78,0.95)$ & $0.32(0.24,0.40)$ \\
\hline Genome & $0.89(0.33,0.97)$ & $0.89(0.20,0.96)$ & $0.90(0.43,0.97)$ & $0.90(0.42,0.97)$ & $0.61(0.24,0.92)$ \\
\hline No. chr w/avg IC $<0.8$ & 1 & 1 & 0 & 0 & 23 \\
\hline No. chr w/min IC $<0.7$ & 2 & 2 & 8 & 10 & 23 \\
\hline
\end{tabular}

mosome 6 . This result could have occurred for many reasons. The IC is lower in this region ( $\mathrm{IC}=0.81$ at peak marker). In addition, because the peak marker does not map uniquely in the build 34 genome assembly, the genetic map position may not be accurate since the genetic map position is linked to physical map position through linear interpolation in this SNP panel. Finally, this result could be a false-positive result in the Illumina mapping panel.

The analysis of STR markers in the same pedigree set resulted in only 3 linkage peaks with corresponding $p$-values $<0.01$. Two of these regions $(2 \mathrm{p} 14,11 \mathrm{q} 23)$ over- lapped with the SNP linkage results and the third region (7p14) did not overlap although it was between two of the SNP linkage peaks (7p22, 7q21). In addition, the IC using the STR data was approximately 3-21\% lower at the linkage peaks compared with the high density SNP data.

\section{Conclusion}

The results on this study suggest that LD between loci in a linkage analysis does not significantly affect the overall detection of linkage regions in a genome scan. However, this result is dependent on the number of genotyped founders or unaffected siblings in the pedigrees because the potential bias in LOD score due to underlying LD

Table 3: Linkage results using the COGA STR marker set (regions with peak $p$-values $<0.0$ I)

\begin{tabular}{|c|c|c|c|c|c|c|c|}
\hline Chromosome & Marker & Position (cM) & Build34 bp & NPL & LOD & $p$-value & IC \\
\hline $2 p \mid 4$ & D2S285 & 94.2 & 67620904 & 2.56 & 1.42 & 0.0070 & 0.90 \\
\hline $7 p \mid 4$ & D7S2846 & 56.8 & 37875515 & 2.67 & 1.55 & 0.0052 & 0.86 \\
\hline 1 Iq23 & DIISI998 & 119.8 & II 7235383 & 2.61 & 1.47 & 0.0062 & 0.69 \\
\hline
\end{tabular}


between SNP loci in a linkage analysis is largest in pedigrees in which genotypes are not available for founding individuals. Therefore, one strategy to determine the effect that LD has on a linkage analysis in other pedigree collections might be to re-run a linkage analysis using a subset of loci with weak or no LD in the pedigree collection being studied. Therefore, one can determine if a linkage result is being inflated by underlying LD between SNP loci in the linkage peak. Despite the potential bias of a resulting LOD score, the high-density SNP panels provide an order of magnitude higher data quality compared to STR genotyping methods and also provide higher IC. In this study, several new linkage regions may have been identified that were not detected using a 10-cM STR marker panel due to higher IC and data quality in the dense SNP linkage panels.

\section{Abbreviations \\ COGA: Collaborative Study of the Genetic of Acoholism}

IC: Information Content

LD: Linkage disequilibrium

NPL: Nonparametric linkage

STR: Short tandem repeat marker

SNP: Single-nucleotide polymorphism

\section{Acknowledgements}

I would like to thank Arnold Oliphant, David Barker, Semyon Kruglyak, and Pauline $\mathrm{Ng}$ for helpful discussions and review of the manuscript.

\section{References}

I. Gyapay G, Morissette J, Vignal A, Dib C, Fizames C, Millasseau P, Marc S, Bernardi G, Lathrop M, Weissenbach J: The 1993-94 Genethon human genetic linkage map. Nat Genet 1994, 7:246-339.

2. Murray JC, Buetow KH, Weber JL, Ludwigsen S, Scherpbierheddema T, Manion F, Quillen J, Sheffield VC, Sunden S, Duyk GM, Weissenbach J, Gyapay G, Dib C, Morrissette J, Lathrop GM, Vignal A, White R, Matsunami N, Gerken S, Melis R, Albertsen H, Plaetke R, Odelberg $S$, Ward D, Dausset J, Cohen D, Cann H: A comprehensive human linkage map with centimorgan density. Cooperative Human Linkage Center (CHLC). Science 1994, 265:2049-2054.

3. Dib C, Faure S, Fizames C, Samson D, Drouot N, Vignal A, Millasseau $P$, Marc S, Hazan J, Seboun E: A comprehensive genetic map of the human genome based on 5,264 microsatellites. Nature 1996, 380:152-154.

4. Broman KW, Murray JC, Sheffield VC, White RL, Weber JL: Comprehensive human genetic maps: individual and sex-specific variation in recombination. Am J Hum Genet 1998, 63:86I-869.

5. Kong A, Gudbjartsson DF, Sainz J, Jonsdottir GM, Gudjonsson SA, Richardsson B, Sigurdardottir S, Barnard J, Hallbeck B, Masson G, Shlien A, Palsson ST, Frigge ML, Thorgeirsson TE, Gulcher JR, Stefansson $\mathrm{K}$ : A high-resolution recombination map of the human genome. Nat Genet 2002, 3 I:24I-247.

6. Weber JL: Human DNA polymorphisms and methods of analysis. Curr Opin Biotechnol 1990, I:166-171.

7. Matise TC, Sachidanandam R, Clark AG, Kruglyak L, Wijsman E, Kakol J, Buyske S, Chui B, Cohen P, de Toma C, Ehm M, Glanowski S, $\mathrm{He}$ C, Heil J, Markianos K, McMullen I, Pericak-Vance MA, Silbergleit A, Stein L, Wagner M, Wilson AF, Winick JD, Winn-Deen ES, Yamashiro CT, Cann HM, Lai E, Holden AL: A 3.9-centimorgan-resolu- tion human single-nucleotide polymorphism linkage map and screening set. Am J Hum Genet 2003, 73:27I-284.

8. Matsuzaki H, Loi H, Dong S, Tsai YY, Fang J, Law J, Di X, Liu WM, Yang G, Liu G, Huang J, Kennedy GC, Ryder TB, Marcus GA, Walsh PS, Shriver MD, Puck JM, Jones KW, Mei R: Parallel genotyping of over 10,000 SNPs using a one-primer assay on a high-density oligonucleotide array. Genome Res 2004, 14:4|4-425.

9. Murray SS, Oliphant A, Shen R, McBride C, Steeke RJ, Shannon SG, Rubano T, Kermani BG, Fan JB, Chee MS, Hansen MS: A highly informative SNP linkage panel for human genetic studies. Nat Meth 2004, I:I I3-I I7.

10. Reich T, Edenberg HJ, Goate A, Williams JT, Rice JP, Van Eerdewegh P, Foroud T, Hesselbrock V, Schuckit MA, Bucholz K, Porjesz B, Li TK, Conneally PM, Nurnberger JI Jr, Tischfield JA, Crowe RR, Cloninger CR, Wu W, Shears S, Carr K, Crose C, Willig C, Begleiter H: Genome-wide search for genes affecting the risk for alcohol dependence. Am J Med Genet 1998, 81:207-2 I5.

II. Slatkin M, Excoffier L: Testing for linkage disequilibrium in genotypic data using the expectation-maximization algorithm. Heredity 1996, 76:377-383.

12. Abecasis GR, Cookson WO: GOLD - graphical overview of linkage disequilibrium. Bioinformatics 2000, 16:182-183.

13. Markianos K, Daly MJ, Kruglyak L: Efficient multipoint linkage analysis through reduction of inheritance space. Am J Hum Genet 200I, 68:963-977.

14. Clerget-Darpoux F: Bias of the estimated recombination fraction and LOD score due to an association between a disease gene and a marker gene. Ann Hum Genet 1982, 46:363-372.

15. Ott J: Strategies for characterizing highly polymorphic markers in human gene mapping. Am J Hum Genet 1992, 5 I:283-290.
Publish with BioMed Central and every scientist can read your work free of charge

"BioMed Central will be the most significant development for disseminating the results of biomedical research in our lifetime. " Sir Paul Nurse, Cancer Research UK

Your research papers will be:

- available free of charge to the entire biomedical community

- peer reviewed and published immediately upon acceptance

- cited in PubMed and archived on PubMed Central

- yours - you keep the copyright

Submit your manuscript here:

http://www.biomedcentral.com/info/publishing_adv.asp
BioMedcentral 